\title{
Advanced patient age is associated with RTT inferior cancer-specific survival after radical nephroureterectomy
}

\author{
Shahrokh F. Shariat ${ }^{1}$, Guilherme Godoy ${ }^{1}$, Yair Lotan ${ }^{1}$, Michael Droller',
} Pierre I. Karakiewicz ${ }^{3}$, Jay D. Raman ${ }^{4}$, Hendrik Isbarn ${ }^{2}$, Alon Weizer ${ }^{5}$, Mesut Remzi ${ }^{6}$, Marco Roscigno ${ }^{7}$, Eiji Kikuchi ${ }^{8}$, Christian Bolenz ${ }^{9}$, Karim Bensalah ${ }^{10}$, Theresa M. Koppie ${ }^{11}$, Wassim Kassouf ${ }^{12}$, Jeffrey C. Wheat ${ }^{5}$, Richard Zigeuner $^{13}$, Cord Langner ${ }^{13}$, Christopher G. Wood ${ }^{14}$ and Vitaly Margulis ${ }^{14}$

${ }^{1}$ University of Texas Southwestern, Dallas, TX, ${ }^{14} \mathrm{MD}$ Anderson Cancer Center, Houston, TX, ${ }^{2}$ Mount Sinai Medical School, ${ }^{4}$ Cornell University, New York, NY, ${ }^{5}$ University of Michigan, Ann Arbor, MI, ${ }^{11}$ University of California Davis, Sacramento, CA, USA, ${ }^{3}$ University of Montreal, ${ }^{12}$ McGill University, Montreal, Quebec, Canada, ${ }^{13}$ Medical University Graz, Graz, ${ }^{6}$ Medical University of Vienna, Vienna, Austria, ${ }^{7}$ Vita-Salute University, Milan, Italy, ${ }^{8}$ Keio University School of Medicine, Tokyo, Japan, ${ }^{9}$ Mannheim Medical Center, University of Heidelberg, Germany, and ${ }^{10}$ University of Rennes, Rennes, France

Accepted for publication 12 August 2009

S.F.S. and G.G. are currently at Memorial Sloan-Kettering Cancer Center in New York, NY, USA

Study Type - Prognosis (case series) Level of Evidence 4

\section{OBJECTIVE}

To assess the impact of patient age on outcomes after radical nephroureterectomy (RNU) for upper tract urothelial carcinoma (UTUC).

\section{PATIENTS AND METHODS}

Data were collected on 1453 patients treated with RNU at 13 centres. Pathological slides were reviewed by dedicated genitourinary pathologists according to standardized criteria. Age at RNU was analysed both as a continuous and categorical variable $(<50$, $n=85 ; 50-59.9, n=229 ; 60-69.9, n=416$ 70-79.9, $n=523$; $\geq 80$ years, $n=200$ ).

\section{RESULTS}

Patients aged $<50$ years were less likely to have undergone previous ureteroscopy and to have a history of bladder cancer $(P \leq 0.026)$. Advanced age was associated with infiltrative architecture and female gender $(P \leq 0.003)$. Patients aged $>70$ years were less likely to undergo lymphadenectomy and to receive adjuvant chemotherapy ( $P \leq 0.026)$. In multivariable analyses, being older was associated with decreased all-cause (AC) survival ( $>60$ years) and cancer-specific survival (CSS; $>80$ years) after controlling for the effects of standard pathological features $(P \leq 0.006)$. However, addition of age did not improve the predictive accuracy of a base model that included standard pathological features for prediction of either disease recurrence, AC survival or CSS.

\section{CONCLUSIONS}

Being older at the time of RNU was associated with decreased survival. This finding could be due to a change in the biological potential of the tumour cell, a decrease in the host's defence mechanisms, or differences in care patterns. Further work is needed to improve our understanding of UTUC outcomes in this growing segment of the population and to develop strategies to improve cancer control in the elderly.

\section{KEYWORDS}

age, prognosis, urinary tract cancer, urothelial carcinoma, survival
INTRODUCTION

RNU is the gold standard treatment for highrisk upper tract urothelial carcinoma (UTUC), and improvements in perioperative care together with refinements of surgical technique have made this a safe and feasible operation for an increasing number of patients. To our knowledge, the extent to which advanced chronological age affects the indications for and outcomes of radical nephroureterectomy (RNU) has not been investigated. Moreover, the effectiveness and relative use of RNU in the subgroup of older patients remain controversial. Similar to bladder cancer, patients might be denied the opportunity to receive the most effective treatment for their disease, based on chronological age alone [1]. In this context, we evaluated the association of patient age with pathological and long-term oncological 
outcomes in a large international cohort of patients treated with RNU for UTUC.

\section{PATIENTS AND METHODS}

This was an Institutional Review Boardapproved study with participating sites providing necessary institutional datasharing agreements before initiation of the study. The international collaboration resulted from the joined efforts of all involved institutions with the main purpose of creating a comprehensive quality data repository with the potential to be explored widely to seek satisfactory answers exploring many different research questions on the diagnosis, prognostic factors and management of UTUC. In all, 13 academic centres worldwide provided data. A computerized databank was generated for data transfer. After combining the data sets, reports were generated for each variable to identify data inconsistencies and other data integrity problems. Through regular communication with all sites, resolution of identified anomalies was achieved before analysis. Before final analysis, the database was frozen and the final data set was produced for the present analysis.

The present study comprised 1453 patients who underwent RNU with ipsilateral bladder cuff resection between 1987 and 2007. Regional lymphadenectomy was generally performed in patients with suspicious lymph nodes on preoperative imaging or with adenopathies detected during intraoperative examination. Extended lymphadenectomy was not routinely performed. Neoadjuvant and adjuvant chemotherapy regimens were administered to 42 (2.9\%) and 169 (11.6\%) patients, respectively.

\section{All surgical specimens were processed} according to standard pathological procedures, and all slides were reviewed by dedicated genitourinary pathologists according to prospectively defined identical criteria. Pathologists were 'blinded' to clinical outcomes. All specimens were histologically confirmed to be UC. Tumours were staged according to the American Joint Cancer Committee/Union Internationale Contre le Cancer TNM classification. Tumour grading was assessed according to the $1998 \mathrm{WHO}$ International Society of Urologic Pathology consensus classification [2]. Lymphovascular invasion was defined as the presence of tumour cells within an endothelium-lined space without underlying muscular walls. The presence of concomitant carcinoma in situ and the tumour architecture were also assessed in every representative section. Tumour necrosis was defined as the presence of microscopic coagulative necrosis whereas the presence of necrosis on gross examination was not included. The extent of tumour necrosis was assessed in a semi-quantitative manner at low magnification (reduced from $\times 40)$. Tumour necrosis was recorded as absent or focally present (0-10\% of tumour area) or extensively present $(>10 \%$ of the tumour area).

Patients were generally followed every 3-4 months for the first year after RNU, every 6 months from the second through to the fifth year, and annually thereafter. Follow-up consisted of history, physical examination, routine blood work and serum chemistry studies, urinary cytology, chest radiography, cystoscopic evaluation of the urinary bladder, and radiographic evaluation of the contralateral upper urinary tract. Elective bone scans, chest $\mathrm{CT}$, or MRI were performed when clinically indicated.

\section{Disease recurrence was defined as local}

failure in the operative site, regional lymph nodes, or distant metastasis. Isolated bladder cancer was not considered as disease recurrence. Cause of death was determined by the treating physicians, by chart review corroborated by death certificates, or by death certificates alone. Death certificates were retrieved from the archived death certificates and reviewed for cause of death. Attribution of cause of death on the death certificate is described in two parts. Part I lists death caused by (i) immediate cause of death (final disease or condition resulting in death) or by (ii) underlying cause of death, and part II lists other significant conditions contributing to death but not resulting in the underlying cause given in part I. Information abstracted from each death certificate included the date of death, and whether UC was noted in either part I or II. To reduce bias in attribution of cause of death, for the present study purposes, only patients who had UC listed in part I of the death certificate were considered to have died of UTUC. All patients who were coded as dead of cancer had previous disease recurrence. Perioperative mortality (any death $\leq 30$ days of surgery or before discharge) was censored at time of death for cancer-specific (CS) mortality analyses.
The Fisher's exact test and the chi-square test were used to evaluate the differences between categorical variables. Differences in age across dichotomous or ranked categories were assessed using the Mann-Whitney $U$ and the Kruskal-Wallis tests, respectively. Univariate recurrence and survival probabilities after RNU were estimated using the Kaplan-Meier method and compared using the log-rank statistic. Univariate and multivariable regression using Cox proportional hazards ratio models addressed time to recurrence, all-cause (AC) mortality, and CS mortality after RNU. In all models, proportional hazards assumptions were systematically verified using the GrambschTherneau residual-based test. Predictive accuracy was quantified with Harrell's concordance index $[3,4]$. Internal validation was performed using 200 bootstrap resamples $[3,4]$. All reported $P$ values were twosided and statistical significance was set at 0.05 . Nominal $P$ values are given with no adjustments made for the calculated values.

\section{RESULTS}

\section{ASSOCIATION OF AGE WITH CLINICAL AND PATHOLOGICAL CHARACTERISTICS}

The median (range; interquartile range [IOR]) interpatient age was $69.7(27-97 ; 15)$ years. At the time of RNU, 85 (5.8\%), 229 (15.8\%), 416 (28.6\%), 523 (36.0\%) and 200 (13.8\%) patients were aged $<50,50-59.9,60-69.9$, 70-79.9, and $>80$ years, respectively. The association of age at RNU with clinicopathological characteristics is shown in Table 1. Being older was significantly associated with a history of previous ureteroscopic intervention, history of bladder cancer, laparoscopic RNU, infiltrative tumour architecture, worse Eastern Cooperative Oncology Group (ECOG) performance status, Caucasian ethnicity, and female gender (all $P \leq 0.02$ ). Lymphadenectomy was performed more commonly in younger patients $(P \leq 0.001)$. Adjuvant chemotherapy was given more commonly to younger patients $(P \leq 0.001)$. There was no association between age at time of RNU and preoperative chemotherapy.

\section{ASSOCIATION BETWEEN AGE AS CONTINUOUS AND CATEGORICAL VARIABLES AND DISEASE OUTCOMES}

The mean (median; range; IQR) follow-up was $61(48 ; 0-250 ; 61)$ months for patients alive 
SHARIAT ET AL.

TABLE 1 Association of age at the time of RNU with clinical and pathological characteristics in 1453 patients treated for UTUC

\begin{tabular}{|c|c|c|c|c|c|c|c|c|}
\hline \multirow[b]{2}{*}{ Variable } & \multirow{2}{*}{$\begin{array}{l}\text { No. patients } \\
(\%)\end{array}$} & \multicolumn{2}{|c|}{$\begin{array}{l}\text { Age (years) as continuous } \\
\text { variable }\end{array}$} & \multicolumn{4}{|c|}{ Age (years) stratified in categories, $n(\%)$} & \multirow[b]{2}{*}{$\geq 80$} \\
\hline & & Median (range) & $P$ & $\overline{<50}$ & $50-59.9$ & $60-69.9$ & $70-79.9$ & \\
\hline$\overline{\text { All }}$ & $1453(100)$ & $69.7(27.0-97.1)$ & - & $85(5.8)$ & 229 (15.8) & 416 (28.6) & $523(36.0)$ & 200 (13.8) \\
\hline Gender: & & & $<0.001^{*}$ & & & & & \\
\hline Male & $986(67.9)$ & $68.1(27.5-97.1)$ & & $61(71.8)$ & $168(73.4)$ & $308(74.0)$ & $334(63.9)$ & $115(57.5)$ \\
\hline Female & $467(32.1)$ & $72.3(27.0-94.3)$ & & 24 (28.2) & $61(26.6)$ & $108(26.0)$ & 189 (36.1) & $85(42.5)$ \\
\hline pT stage: & & & $0.281+$ & & & & & \\
\hline Ta & $295(20.3)$ & $70.2(31.0-94.3)$ & & $20(23.5)$ & $48(21.0)$ & $73(17.5)$ & $118(22.6)$ & $36(18.0)$ \\
\hline Tis & $28(1.9)$ & $67.0(32.0-86.1)$ & & $1(1.2)$ & $5(2.2)$ & $12(2.9)$ & $7(1.3)$ & $3(1.5)$ \\
\hline $\mathrm{T} 1$ & $317(21.8)$ & $68.0(34.1-90.7)$ & & $20(23.5)$ & $57(24.9)$ & 95 (22.8) & $100(19.1)$ & $45(22.5)$ \\
\hline T2 & $269(18.5)$ & $69.2(30.0-96.9)$ & & $16(18.8)$ & $47(20.5)$ & $75(18.0)$ & $92(17.6)$ & $39(19.5)$ \\
\hline T3 & $475(32.7)$ & $70.0(27.0-97.1)$ & & $26(30.6)$ & $62(27.1)$ & $145(34.9)$ & $179(34.2)$ & $63(31.5)$ \\
\hline T4 & $69(4.7)$ & $72.9(43.0-89.0)$ & & $2(2.4)$ & $10(4.4)$ & $16(3.8)$ & $27(5.2)$ & $14(7.0)$ \\
\hline History of bladder cancer: & & & $0.003^{*}$ & & & & & \\
\hline Absent & $1085(74.7)$ & $69.0(27.0-96.9)$ & & $74(87.1)$ & 177 (77.3) & $314(75.5)$ & $379(72.5)$ & $141(70.5)$ \\
\hline Present & $368(25.3)$ & $71.0(40.0-97.1)$ & & $11(12.9)$ & $52(22.7)$ & $102(24.5)$ & $144(27.5)$ & $59(29.5)$ \\
\hline Previous endoscopic procedures: & & & $0.023^{*}$ & & & & & \\
\hline Negative & $1253(86.2)$ & $69.0(27.0-97.1)$ & & 78 (91.8) & $203(88.6)$ & $361(86.8)$ & $441(84.3)$ & $170(85.0)$ \\
\hline Positive & $200(13.8)$ & $71.0(31.0-94.3)$ & & $7(8.2)$ & $26(11.4)$ & 55 (13.2) & $82(15.7)$ & $30(15.0)$ \\
\hline Tumour grade: & & & $0.198^{*}$ & & & & & \\
\hline Low & $516(35.5)$ & $69.4(30.0-96.9)$ & & $37(43.5)$ & $85(37.1)$ & $142(34.1)$ & $185(35.4)$ & $67(33.5)$ \\
\hline High & $937(64.5)$ & $70.0(27.0-97.1)$ & & $48(56.5)$ & $144(62.9)$ & $274(65.9)$ & $338(64.6)$ & $133(66.5)$ \\
\hline Lymph nodes status: & & & $<0.001+$ & & & & & \\
\hline $\mathrm{pNx}$ & $841(57.9)$ & $70.9(27.5-97.1)$ & & 44 (51.8) & $121(52.0)$ & $224(53.8)$ & $315(60.2)$ & $137(68.5)$ \\
\hline pNO & $472(32.5)$ & $68.0(30.7-96.9)$ & & $29(34.1)$ & 89 (38.9) & $147(35.3)$ & $162(31.0)$ & $45(22.5)$ \\
\hline $\mathrm{pN} 1-2$ & $140(9.6)$ & $68.0(27.0-90.0)$ & & $12(14.1)$ & $19(8.3)$ & 45 (10.8) & 46 (8.8) & $18(9.0)$ \\
\hline ECOG performance status: & & & $<0.001+$ & & & & & \\
\hline 0 & $1001(68.9)$ & $68.0(27.0-91.0)$ & & 67 (78.8) & 177 (77.3) & $308(74.0)$ & $348(66.5)$ & $101(50.5)$ \\
\hline 1 & $358(24.6)$ & $72.0(30.7-96.9)$ & & 15 (17.6) & 44 (19.2) & $85(20.4)$ & $137(26.2)$ & $77(38.5)$ \\
\hline 2 & $85(5.8)$ & $74.4(37.3-90.0)$ & & $2(2.4)$ & $7(3.1)$ & $20(4.8)$ & $36(6.9)$ & $20(10.0)$ \\
\hline 3 & $9(0.6)$ & $64.1(48.6-97.1)$ & & $1(1.2)$ & $1(0.4)$ & $3(0.7)$ & $2(0.4)$ & $2(1.0)$ \\
\hline Ethnicity: & & & $0.004 t$ & & & & & \\
\hline White & $1194(82.2)$ & $70.1(27.0-97.1)$ & & $66(77.6)$ & $175(76.4)$ & 341 (82.0) & 439 (83.9) & $173(86.5)$ \\
\hline Black & $36(2.5)$ & $69.5(30.0-85.0)$ & & $6(7.1)$ & $4(1.7)$ & $8(1.9)$ & $10(1.9)$ & $8(4.0)$ \\
\hline Pacific Islander/Asian & $223(15.3)$ & $67.0(36.0-89.0)$ & & $13(15.3)$ & $50(21.8)$ & $67(16.1)$ & $74(14.1)$ & $19(9.5)$ \\
\hline Lymphovascular invasion: & & & $0.063^{*}$ & & & & & \\
\hline Absent & 1104 (76) & $69.0(30.0-97.1)$ & & $64(75.3)$ & $186(81.2)$ & $319(76.7)$ & $383(73.2)$ & $152(76.0)$ \\
\hline Present & $349(24)$ & $70.6(27.0-94.0)$ & & $21(24.7)$ & 43 (18.8) & 97 (23.3) & $140(26.8)$ & $48(24.0)$ \\
\hline Architecture: & & & $<0.001^{*}$ & & & & & \\
\hline Papillary & 1066 (73.4) & $68.7(27.0-97.1)$ & & 72 (84.7) & $180(78.6)$ & $313(75.2)$ & $358(68.5)$ & $143(71.5)$ \\
\hline Infiltrative & 387 (26.6) & $71.4(34.1-91.0)$ & & $13(15.3)$ & 49 (21.4) & 103 (24.8) & 165 (31.5) & 57 (28.5) \\
\hline Procedure type: & & & $0.006^{*}$ & & & & & \\
\hline Open & 1128 (77.6) & $69.0(27.0-97.1)$ & & 64 (75.3) & 185 (80.8) & 341 (82.0) & 397 (75.9) & 141 (70.5) \\
\hline Laparoscopic & 325 (22.4) & $71.1(32.0-94.3)$ & & $21(24.7)$ & 44 (19.2) & 75 (18.0) & $126(24.1)$ & 59 (29.5) \\
\hline Tumour necrosis: & & & $0.278^{*}$ & & & & & \\
\hline Absent & 1089 (74.9) & $69.5(27.0-96.9)$ & & 65 (76.5) & 179 (78.2) & $310(74.5)$ & 386 (73.8) & $149(74.5)$ \\
\hline Present & $364(25.1)$ & $70.0(37.3-97.1)$ & & $20(23.5)$ & 50 (21.8) & $106(25.5)$ & 137 (26.2) & 51 (25.5) \\
\hline Tumour location: & & & $0.107^{*}$ & & & & & \\
\hline Renal pelvis & $958(65.9)$ & $69.0(27.0-96.9)$ & & 64 (75.3) & 158 (69.0) & $271(65.1)$ & 330 (63.1) & $135(67.5)$ \\
\hline Ureter & $495(34.1)$ & $70.3(27.5-97.1)$ & & $21(24.7)$ & 71 (31.0) & 145 (34.9) & 193 (36.9) & 65 (32.5) \\
\hline Concomitant carcinoma in situ: & & & $0.237^{*}$ & & & & & \\
\hline Negative & $1035(71.2)$ & $69.1(27.0-97.1)$ & & 67 (78.8) & $171(74.7)$ & $289(69.5)$ & $369(70.6)$ & $139(69.5)$ \\
\hline Positive & $418(28.8)$ & $70.0(32.0-93.0)$ & & $18(21.2)$ & $58(25.3)$ & $127(30.5)$ & $154(29.4)$ & 61 (30.5) \\
\hline Adjuvant chemotherapy: & & & $<0.001^{*}$ & & & & & \\
\hline Negative & $1249(86.0)$ & $70.0(27.5-97.1)$ & & $70(82.4)$ & $193(84.3)$ & $346(83.2)$ & $456(87.4)$ & $184(92.0)$ \\
\hline Positive & $203(14.0)$ & $66.0(27.0-93.0)$ & & $15(17.6)$ & $36(15.7)$ & $70(16.8)$ & 66 (12.6) & $16(8.0)$ \\
\hline
\end{tabular}

*Mann-Whitney test; +Kruskal-Wallis test. 
TABLE 2 Univariate and multivariable Cox regression analyses predicting disease recurrence, AC survival and CSS in 1453 patients treated with RNU for UTUC

\begin{tabular}{|c|c|c|c|c|c|c|c|c|c|c|c|c|}
\hline \multirow[b]{3}{*}{ Variable } & \multicolumn{4}{|c|}{ Disease recurrence } & \multicolumn{4}{|c|}{$A C$ survival } & \multicolumn{4}{|l|}{ CSS } \\
\hline & \multicolumn{2}{|c|}{ Univariate } & \multicolumn{2}{|c|}{ Multivariable } & \multicolumn{2}{|c|}{ Univariate } & \multicolumn{2}{|c|}{ Multivariable } & \multicolumn{2}{|c|}{ Univariate } & \multicolumn{2}{|c|}{ Multivariable } \\
\hline & $\overline{\mathrm{HR}}$ & $P$ & $\overline{\mathrm{HR}}$ & $P$ & $\overline{\mathrm{HR}}$ & $P$ & $\overline{\mathrm{HR}}$ & $P$ & $\overline{\mathrm{HR}}$ & $P$ & $\overline{\mathrm{HR}}$ & $P$ \\
\hline$\overline{\text { pT stage }}$ & & $<0.001$ & & $<0.001$ & & $<0.001$ & & $<0.001$ & & $<0.001$ & & $<0.001$ \\
\hline $\mathrm{T} 1$ vs $\mathrm{Ta} / \mathrm{Tis}$ & 2.85 & $<0.001$ & 2.14 & 0.02 & 1.45 & 0.03 & 1.21 & 0.3 & 2.75 & 0.004 & 2.13 & 0.030 \\
\hline $\mathrm{T} 2$ vs $\mathrm{Ta} / \mathrm{Tis}$ & 6.76 & $<0.001$ & 4.62 & $<0.001$ & 2.37 & $<0.001$ & 1.96 & $<0.001$ & 7.16 & $<0.001$ & 5.10; & $<0.001$ \\
\hline $\mathrm{T} 3$ vs $\mathrm{Ta} / \mathrm{Tis}$ & 15.28 & $<0.001$ & 8.66 & $<0.001$ & 4.19 & $<0.001$ & 3.13 & $<0.001$ & 16.31 & $<0.001$ & 9.82 & $<0.001$ \\
\hline $\mathrm{T} 4 \mathrm{vs} \mathrm{Ta} / \mathrm{Tis}$ & 52.36 & $<0.001$ & 21.34 & $<0.001$ & 12.28 & $<0.001$ & 6.58 & $<0.001$ & 52.55 & $<0.001$ & 21.28 & $<0.001$ \\
\hline LVI & 3.31 & $<0.001$ & 1.29 & 0.03 & 2.57 & $<0.001$ & 1.40 & 0.002 & 3.55 & $<0.001$ & 1.38 & 0.01 \\
\hline Tumour grade & 5.15 & $<0.001$ & 2.50 & $<0.001$ & 2.37 & $<0.001$ & 1.56 & $<0.001$ & 4.72 & $<0.001$ & 2.20 & $<0.001$ \\
\hline Lymph node status & & $<0.001$ & & $<0.001$ & & $<0.001$ & & $<0.001$ & & $<0.001$ & & $<0.001$ \\
\hline Nx vs NO & 1.09 & 0.5 & 1.54 & $<0.001$ & 1.37 & 0.002 & 1.61 & $<0.001$ & 1.21 & 0.2 & 1.65 & $<0.001$ \\
\hline Npositive vs NO & 4.97 & $<0.001$ & 2.29 & $<0.001$ & 3.46 & $<0.001$ & 1.77 & $<0.001$ & 5.03 & $<0.001$ & 2.21 & $<0.001$ \\
\hline Age & 1.01 & 0.054 & 1.01 & 0.3 & 1.03 & $<0.001$ & 1.03 & $<0.001$ & 1.02 & $<0.001$ & 1.02 & 0.004 \\
\hline
\end{tabular}

LVI, Iymphovascular invasion; Nx, lymphadenectomy not performed.

\begin{tabular}{|c|c|c|c|c|}
\hline \multirow[b]{2}{*}{ Variable } & \multicolumn{2}{|c|}{ Univariate analysis } & \multicolumn{2}{|c|}{$\begin{array}{l}\text { Multivariable analysis } \\
\text { with age as categorical } \\
\text { variable }\end{array}$} \\
\hline & $\overline{\mathrm{HR}}$ & $P$ & $\overline{\mathrm{HR}}$ & $P$ \\
\hline Age predicting $A C$ survival & & $<0.001$ & & $<0.001$ \\
\hline $50-59$ vs $\leq 50$ years & 0.97 & 0.9 & 0.92 & 0.7 \\
\hline $60-69$ vs $\leq 50$ years & 1.57 & 0.04 & 1.52 & 0.06 \\
\hline $70-79$ vs $\leq 50$ years & 1.93 & 0.003 & 1.78 & 0.01 \\
\hline$\geq 80$ vs $\leq 50$ years & 2.68 & $<0.001$ & 2.51 & $<0.001$ \\
\hline Age predicting CSS & & 0.02 & & $<0.001$ \\
\hline $50-59$ vs $\leq 50$ years & 0.85 & 0.6 & 0.81 & 0.5 \\
\hline $60-69$ vs $\leq 50$ years & 1.30 & 0.3 & 1.30 & 0.3 \\
\hline $0-79$ vs $\leq 50$ years & 1.19 & 0.5 & 1.09 & 0.8 \\
\hline$\geq 80$ vs $\leq 50$ years & 1.69 & 0.03 & 1.63 & 0.04 \\
\hline
\end{tabular}

All models corrected for pT stage, lymphovascular invasion, tumour grade and lymph node status.

at last follow-up. Actuarial recurrence-free estimates at 2, 5, and 10 years after RNU were $73 \%, 69 \%$, and $66 \%$, respectively. Actuarial AC survival estimates at 2, 5, and 10 years after RNU were $73 \%, 61 \%$, and $47 \%$, respectively. Actuarial CS survival (CSS) estimates at 2, 5, and 10 years after RNU were $79 \%, 72 \%$, and 69\%, respectively.

In univariate analyses, age was significantly associated with $\mathrm{AC}$ and CS mortality, but not disease recurrence (Table 2). In univariate Cox regression analyses, being older was associated with increased risk of AC (Table 2, $P<0.001$ ) and CS mortality (Table 2 ,
$P<0.001)$, but not disease recurrence

(Table 2, $P=0.054$ ).

In multivariable analyses controlling for the effects of tumour stage, grade, lymph node status, and lymphovascular invasion, age was an independent predictor of both AC (Table 2, $P<0.001$ ) and CS mortality (Table 2,

$P=0.004)$. Subgroup analysis in patients who did not undergo lymphadenectomy did not change the statistical significance of these associations.

To further investigate the significant relationship between age at the time of
RNU and AC and CS mortality, similar Cox regression analyses were performed controlling for the effects of the same cofactors as the previous models, but including age as a categorical variable. In univariate and multivariable analyses, an age of $\geq 60$ years (univariate $P<0.001$ and multivariable $P<0.001)$ and $\geq 80$ years (univariate $P=0.02$ and multivariable $P<0.001)$ were significantly associated with AC and CS mortality, respectively (Table 3 ).

The predictive accuracy of age as continuous variable for prediction of disease recurrence, AC survival, and CSS was 52.3\%, 59.2\%, and $55.9 \%$, respectively. The predictive accuracies of age as a categorical variable for prediction of $A C$ and CS mortality were $57.9 \%$, and $54.3 \%$, respectively (Fig. 1). The addition of age as categorical variable did not improve the predictive accuracies of the base model that comprised pathological stage, grade, lymphovascular invasion, and lymph node status for prediction of disease recurrence, AC and CS mortality.

\section{DISCUSSION}

Compared with other cancers, there is little data on the impact of age on patterns of care and outcomes of patients with UTUC. In the present study, $6 \%$ of patients treated with RNU were aged $<50$ years and $14 \%$ were aged $>80$ years. Being older was associated with a history of previous ureteroscopy, history of bladder cancer, laparoscopic RNU, infiltrative tumour architecture, worse ECOG performance status and female gender. 
Elderly patients were less likely to undergo lymphadenectomy or receive postoperative chemotherapy for advanced stage disease. Most importantly, age was an independent predictor of AC and CS mortality.

Being older was associated with CS mortality, but not disease recurrence, after controlling for the effects of standard pathological features. Reasons for this could be a change in the biological potential of the tumour cell or a decrease in the host's defence mechanisms with advancing age, or even a detrimental effect of differences in care patterns in elderly compared with younger patients. Indeed, in the present study advanced age was associated with features of advanced disease such as higher rate of infiltrative tumour architecture, which has been shown to be an ominous independent predictor of outcomes after UTUC [5]. In addition, while the rate of $\mathrm{pTa}$ disease decreased, that of pT4 disease increased with advancing age.

The higher CS mortality of elderly patients treated with RNU compared with younger patients may be secondary to suboptimal use of RNU (patient selection and delay), lymphadenectomy, and/or postoperative chemotherapy. It may be that similar to bladder cancer, there is a reluctance to perform radical surgery in older patients with comorbidities resulting in higher tumour stage when surgery is eventually performed leading to higher CS mortality $[1,6]$. Thus, the association between advanced age and worse outcomes in the present study may be a selection bias resulting in treatment with RNU of elderly patients with relatively more advanced disease.

An alternative explanation may be that there is a longer delay between diagnosis and definitive treatment in elderly patients resulting in disease progression and worse survival [7-9]. In support of this, in the present study older patients were more likely to undergo multiple ureteroscopic interventions before the decision to perform a RNU compared with their younger counterparts.

Similar to the reported data on bladder UC, elderly patients with UTUC were less likely to receive postoperative chemotherapy in the presence of advanced disease stage $[1,10]$. This may be partially due to the frailty and presence of significant comorbidities in these
FIG. 1. Probability estimates of (A) recurrence-free survival and (B) CSS in 1453 patients treated with RNU for UTUC stratified by age categorized into <50 (85 patients), 50-59.9 (229), 60-69.9 (416), 70-79.9 (523), and $\geq 80$ years (200).

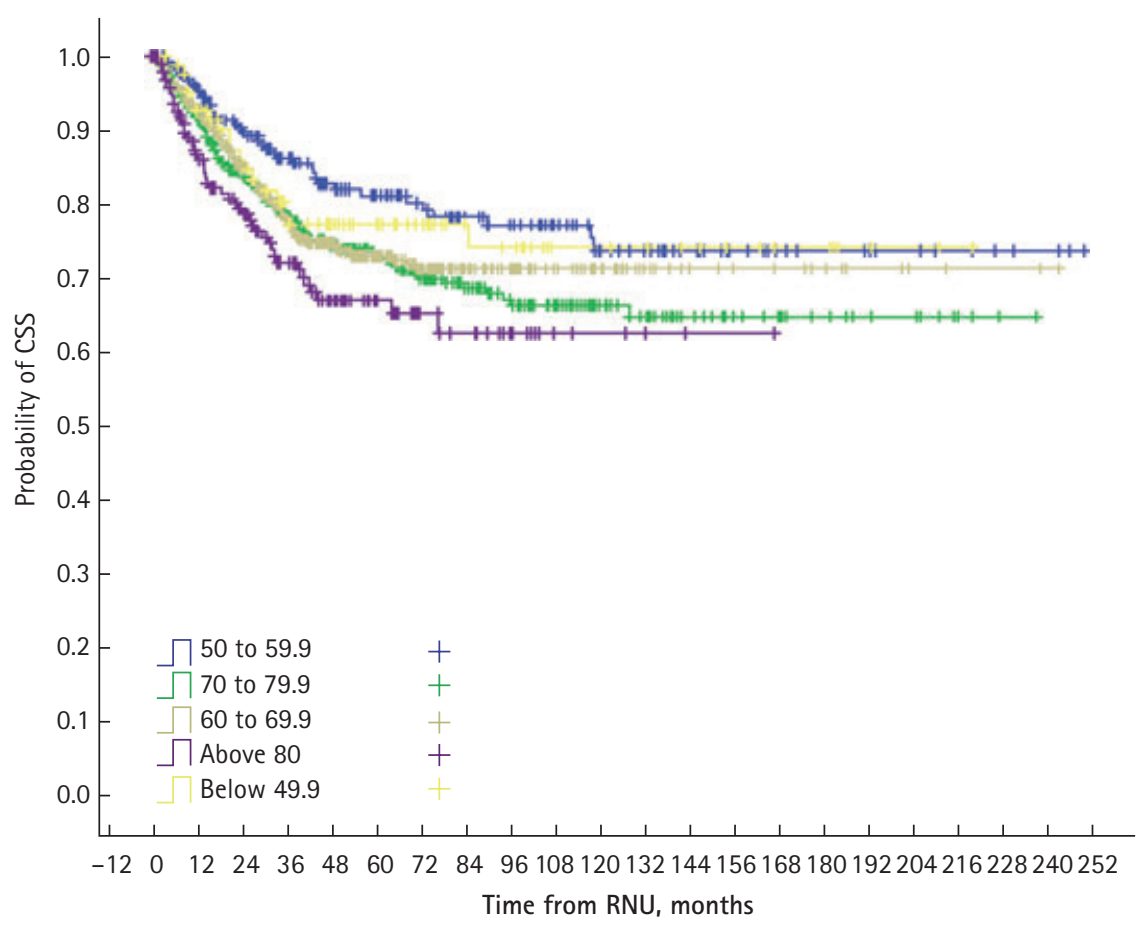

patients, such as impaired renal function and cardiac disease. The risk/benefit ratio is differently weighted in the elderly compared with younger patients. The compromise between life years gained vs quality of life may be different from the perspective of elderly compared with younger patients. Similarly, elderly patients were less likely to undergo lymphadenectomy. We and others have previously shown that nodal status is a significant predictor of CS mortality in UTUC and that $\mathrm{pNx}$ is significantly associated with a worse prognosis than pNO in the subgroup of muscle-invasive tumours [11]. Moreover, it has also been recently shown that the extension of the lymphadenectomy in patients with pNO UTUC seems to be associated with CS mortality [12].

The host response might also play a critical role in the differential CS mortality rates between young and older patients treated with RNU for UTUC. In bladder UC, Miller et al. [13] reported that the Charlson Index score was a strong predictor of increased risk of extravesical disease and CS mortality. Unfortunately, we did not have data to rigorously examine the impact of the comorbidities in the present cohort. Weizer et al. [14] reported that Karnofsky performance status was an independent predictor of overall survival on patients aged $\geq 70$ years presenting with nonmetastatic muscle-invasive bladder UC. Moreover, their study confirmed that most elderly patients were able to undergo definitive treatment supporting the point that in elderly patients with a good performance status, radical surgery offers the best disease control.

There are several limitations in the present study. Our results were analysed retrospectively and did not control for possible selection bias such as presence of patient comorbidities. While there was an association between age and survival endpoints, this association was not true for disease recurrence. This could be explained by the decrease in the host's defence mechanisms with advancing age and/or differences in selection for administration of adjuvant chemotherapy. We are unable to comment on the extent to which patient age factored into the selection of RNU and/or perioperative chemotherapy among available treatment options for patients presenting with high-risk disease. The impact of an agebased selection bias on treatment outcomes 
for UTUC is probably substantial, particularly given the suspected relative under use of RNU for elderly patients. The relative under use of chemotherapy in older patients in the present series suggests a bias against adjuvant treatment in our older patients; the individual reasons for this were not available. A careful analysis of other factors not addressed in the present study, such as the time from invasive UTUC diagnosis to the RNU and the impact of comorbidities is needed to have a better understanding of the effect of age on tumour biology and patient survival. Moreover, we did not have data on functional status outcomes. Such data have not been systematically examined and may add to the perspective of quality-of-life-adjusted relative survival benefits.

\section{Another potential limitation is that the} patients were treated by various physicians and the specimens were evaluated by various pathologists over a long period. Although the differences in practice patterns across the institutions in the present study may affect the interpretation of our findings, they are reflective of the real world scenario, making the conclusions more generalizable. To minimize the difference in pathological evaluation of the specimens, all specimens were reviewed by dedicated genitourinary pathologists according to strict morphological criteria to standardize reproducibility. Furthermore, this is the largest reported series of patients treated with RNU for UTUC and it is unlikely that large prospective randomized trials will be organized, due to the relative rarity of this disease $[15,16]$. The limited predictive accuracy of our models may suggest that age and other important related factors, which could not be explored in the present study, may play an important role in determining the outcomes of this patient population.

In conclusion, the present study suggests that delay in definitive treatment, lack of lymphadenectomy, and potential under use of adjuvant chemotherapy are potential modifiable risk factors that could lead to improvement of outcomes in elderly patients. This together with the efficacy of RNU for disease control suggests that elderly patients with high-risk UTUC who have an adequate performance status should be offered RNU earlier. The limitations of the present study do not allow us to make recommendations based on our data; however, our observations are provocative and deserve further attention to identify the causes for the worse CS mortality in elderly patients and to develop strategies to improve cancer control in this population.

\section{CONFLICT OF INTEREST}

None declared. Source of Funding: National Institute of Health; T32 training grant (T32CA082088).

\section{REFERENCES}

1 Nielsen ME, Shariat SF, Karakiewicz PI et al. Advanced age is associated with poorer bladder cancer-specific survival in patients treated with radical cystectomy. Eur Urol 2007; 51: 699-708

2 Epstein Jl, Amin MB, Reuter VR, Mostofi FK. The World Health Organization/International Society of Urological Pathology consensus classification of urothelial (transitional cell) neoplasms of the urinary bladder. Bladder Consensus Conference Committee. Am J Surg Pathol 1998; 22: 1435-48

3 Harrell FE Jr, Califf RM, Pryor DB, Lee $\mathrm{KL}$, Rosati RA. Evaluating the yield of medical tests. JAMA 1982; 247: 2543-6

4 Harrell FE Jr, Lee KL, Mark DB. Multivariable prognostic models: issues in developing models, evaluating assumptions and adequacy, and measuring and reducing errors. Stat Med 1996; 15: 361-87

5 Remzi M, Haitel A, Margulis V et al. Tumour architecture is an independent predictor of outcomes after nephroureterectomy: a multiinstitutional analysis of 1363 patients. BJU Int 2009; 103: 307-11

6 Prout GR Jr, Wesley MN, Yancik R, Ries LA, Havlik RJ, Edwards BK. Age and comorbidity impact surgical therapy in older bladder carcinoma patients: a population-based study. Cancer 2005; 104: 1638-47

7 Nielsen ME, Palapattu GS, Karakiewicz $\mathrm{PI}$ et al. A delay in radical cystectomy of $>3$ months is not associated with a worse clinical outcome. BJU Int 2007; 100: 1015-20

8 Chang SS, Hassan JM, Cookson MS, Wells N, Smith JA Jr. Delaying radical cystectomy for muscle invasive bladder cancer results in worse pathological stage. J Uro/ 2003; 170: 1085-7

9 Sanchez-Ortiz RF, Huang WC, Mick R, Van Arsdalen KN, Wein AJ, Malkowicz
SB. An interval longer than 12 weeks between the diagnosis of muscle invasion and cystectomy is associated with worse outcome in bladder carcinoma. J Urol 2003; 169: 110-5

10 Clark PE, Stein JP, Groshen SG et al. Radical cystectomy in the elderly: comparison of clincal outcomes between younger and older patients. Cancer 2005; 104: 36-43

11 Roscigno M, Shariat SF, Margulis V et al. Impact of Lymph Node Dissection on Cancer-Specific Survival in Patients with Upper Tract Urothelial Carcinoma Treated with Radical Nephroureterectomy. Eur Urol 2009; 181: 2482-9

12 Roscigno M, Shariat SF, Margulis V et al. The extent of lymphadenectomy seems to be associated with better survival in patients with nonmetastatic upper-tract urothelial carcinoma: how many lymph nodes should be removed? Eur Urol 2009; 56: 512-8

13 Miller DC, Taub DA, Dunn RL, Montie JE, Wei JT. The impact of co-morbid disease on cancer control and survival following radical cystectomy. J Uro/ 2003; 169: 105-9

14 Weizer AZ, Joshi D, Daignault S et al. Performance status is a predictor of overall survival of elderly patients with muscle invasive bladder cancer. J Urol 2007; 177: 1287-93

15 Jemal A, Thun MJ, Ries LA et al. Annual report to the nation on the status of cancer, 1975-2005, featuring trends in lung cancer, tobacco use, and tobacco control. J Natl Cancer Inst 2008; 100: 1672-94

16 Jemal A, Ward E, Hao Y, Thun M. Trends in the leading causes of death in the United States, 1970-2002. JAMA 2005; 294: 1255-9

Correspondence: Shahrokh F. Shariat, Division of Urology; Sidney Kimmel Center for Prostate and Urologic Cancer, Memorial Sloan-Kettering Cancer Center, 1275 York Avenue, Box 27, New York, NY 10065, USA.

e-mail: shariats@mskcc.org; sfshariat@gmail.com

Abbreviations RNU, radical nephroureterectomy; (UT)UC, (upper tract) urothelial carcinoma; $\mathrm{AC}$, all-cause; CS(S), cancer-specific (survival); IOR, interquartile range; ECOG, Eastern Cooperative Oncology Group. 